\title{
The genetic control of meiotic drive acting on the B-chromosome of Myrmeleotettix maculatus (Orthoptera: Acrididae)
}

\author{
M. W. Shaw* and G. M. Hewitt
}

School of Biological Sciences, University of East

Anglia, Norwich, NR4 7TJ

\begin{abstract}
Crosses between populations with and without B-chromosomes were made, and backcrossed to the non $B$ parent for two generations. No polygenic differences in male or female meiotic transmission were found, but a modifier of meiotic drive segregated in the experiment, drastically reducing female transmission rate. We tentatively interpret this as evidence of a coevolutionary race between the $B$-chromosome and the $A$ genome.
\end{abstract}

\section{INTRODUCTION}

The B-chromosome of Myrmeleotettix maculatus is polymorphic in populations of the grasshopper found over a large part of Wales and Southern England (Hewitt, 1973). Outside this area it is absent, although a single population exists off the coast of Sweden with a similar element present at a low frequency (Ramel, 1981). It appears that the B is maintained in those populations in which it is present through the opposing pressures of a large net meiotic drive and strong selection against individuals with two B's (Shaw, 1984). B-chromosomes of many species have accumulation mechanisms (Jones and Rees, 1983), so this is probably a common reason for their presence in a population.

In the present instance, two questions naturally arise. First, what factor or factors control the geographic distribution of the $\mathrm{B}$, limiting it in the way described above? Second, what evolutionary consequences arise from the presence of the $B$ in populations where it is present? The present study reports an experiment which was designed to shed some light on the former question, and incidentally allowed us to glimpse something of the answer to the second.

The geographic distribution of the $B$ could be entirely fortuitous, the result of a finite rate of

\footnotetext{
* Present address: Long Ashton Research Station, University of Bristol, Long Ashton, Bristol, BS18 9AF
}

spread and the origin of the B in a single location. Support for this hypothesis comes from the discovery of forward movement of the cline between $B$ and no-B populations over a period of ten years (Shaw, 1983). On the other hand, the strong correlation between temperature and $B$ frequency within a small area found by Hewitt and Ruscoe (1971) suggests that the overall distribution may be controlled by temperature (Hewitt and John, 1967; Barker, 1966) or some other climatic feature. Environmental factors could act by altering either meiotic drive or the fitness of $1 \mathrm{~B}$ individuals so that the equilibrium B-frequency became zero or less. For example, Shaw and Hewitt (1984) present evidence that at lower temperatures the meiotic transmission rate of some males may be reduced, which would reduce the average net meiotic drive in the population. Alternatively, environmental factors might act indirectly: populations adapted to cooler regimes (or whatever) might, as an incidental pleiotropic effect, have altered transmission rates or fitnesses, causing the $\mathrm{B}$ to be eliminated. For example, Teoh and Jones (1978) demonstrated that meiotic drive varied between stocks of ryegrass with different evolutionary histories.

The presence of a B in a population will favour the increase in frequency of genes which tend to ameliorate its effects in individuals happening to carry it, whether directly or by reducing the frequency with which it is passed to the offspring. 
The selection pressure on such a "modifier" gene varies with B-frequency, and if the modifier has any effects unfavourable to animals without B's a frequency-dependent equilibrium will eventually be reached. This will cause either a heritable continuous distribution of transmission frequencies, or a distinct polymorphism. The processes are discussed in more detail in Shaw (1984) for this particular case and are broadly analogous to those discussed by (for example) Prout, Bundgaard and Bryant (1973).

The present paper is a report of an experiment designed to investigate whether genetic differences in B-chromosome transmission rate could explain its geographic distribution. We did this by backcrossing the $\mathrm{B}$ onto the genetic background of a population without B's. The results revealed no clear geographic differences, but demonstrated a polymorphism in B-chromosome transmission frequency within populations.

\section{MATERIALS AND METHODS}

A sample of grasshoppers some of whose members contained B-chromosomes was collected as 4th (pre-adult) instars from Shakers Furze (OS 913955 TL) in Norfolk, and formed the source of B's for the experiment. A collection of 4 th instars from Winterton (OS $500200 \mathrm{TG}$ ) on the Norfolk coast, some $80 \mathrm{~km}$ distant from the nearest $\mathrm{B}$ containing population, was used as $O B$ parents. Individuals were mated as single pairs in small polypropylene gauze cages supplied with cocksfoot (Dactylis glomerata) as food. Small boxes of dry sand were provided for females to lay eggs in. Pods of eggs were kept in moist sand at lab. temperature $\left(23^{\circ} \mathrm{C}-26^{\circ} \mathrm{C}\right)$ for $4-5$ weeks, then at $4^{\circ} \mathrm{C}$ for 8 weeks or more to break diapause. After 10 days in an incubator at $26^{\circ} \mathrm{C}$, they were hatched by burying them in moist sand surrounding a Dactylis plant growing in a $15 \mathrm{~cm}$ plant pot. This was covered in a clear plastic propagator (Stewarts Plastics Ltd) lengthened with a polypropylene mesh insert to improve ventilation and provide more space for the grass. Survival under these conditions was excellent: up to 100 per cent of individuals survived to 4 th instar; the median survival was around 50 per cent. At 4 th instar individuals were removed to single pair cages. These methods are described more fully in Shaw (1981), and derive via Harvey (1977) from KellyStebbings and Hewitt (1972).

The male partner in a cross was karyotyped, after the female had begun to lay, by vivisecting the testis, fixing it in $3: 1$ ethanol:acetic acid, and squashing a few follicles in lacto-propionic orcein. Eggs were karyotyped either 17 days after laying or 3 days after removal from diapause; both gave good mitotic indices. The embryos were dissected into 0.05 per cent colchocine in insect ringer, left for about 1 hour, then fixed and stained as for the testis material. No attempt was made to karyotype females; their karyotypes were deduced from those of their offspring and their male partner.

\section{Design}

The study of inheritance of meiotic drive in $M$. maculatus poses some problems. The generation time that can be achieved in the laboratory is not much less than six months. The karyotype of an individual cannot be determined until after it has mated, and, in the case of a female, completed egg-laying: thus many useless crosses are inevitably set up. Lastly, meiotic drive in an individual can only be scored in its progeny, so an individual must be reared to adult and successfully crossed before it can be scored.

Bearing in mind these problems, our plan was to cross a few $B$ containing individuals from Shakers Furze with individuals from the Winterton population, where the B is absent. By collecting many eggpods from these crosses the drive of individuals in the parent population could be measured, while leaving enough pods to rear an " $F_{1}$ " generation. This would again be backcrossed to the Winterton stock and many pods collected, so as to allow both the estimation of the " $F_{1}$ " transmission rates and the rearing of individuals which could be further backcrossed to characterise their drive rates.

This design is sufficient to estimate additive and dominance genetic differences in drive and drag between the populations. By using several independently isolated B-chromosomes, differences in transmission rate among these could be estimated. They can be separated from additive genetic effects of the rest of the genome because they should be constant regardless of the degree of relatedness of the animals carrying the B. Systematic differences between generations due to some environmental influence or unconscious selection could be checked by rearing some purebred B containing stock in each generation, alongside the cross-breds, and assessing their transmission rates. However, since labour and space were limited the experiment had to be kept fairly small and its precision was not great: about 250 crosses were made in all. 
The initial crosses were made in two batches. In February and March 1979 stocks were reared from pods laid in crosses by adult females captured in October 1978; in June and July 1979 individuals captured as 4 th instar nymphs from the wild were used. At this time the intrapopulation crosses needed as controls and to provide individuals for backcrossing to were also made. The " $F_{1}$ " generation was reared and backcrosses made in February and March 1980; the second backcross was made in May and June 1981.

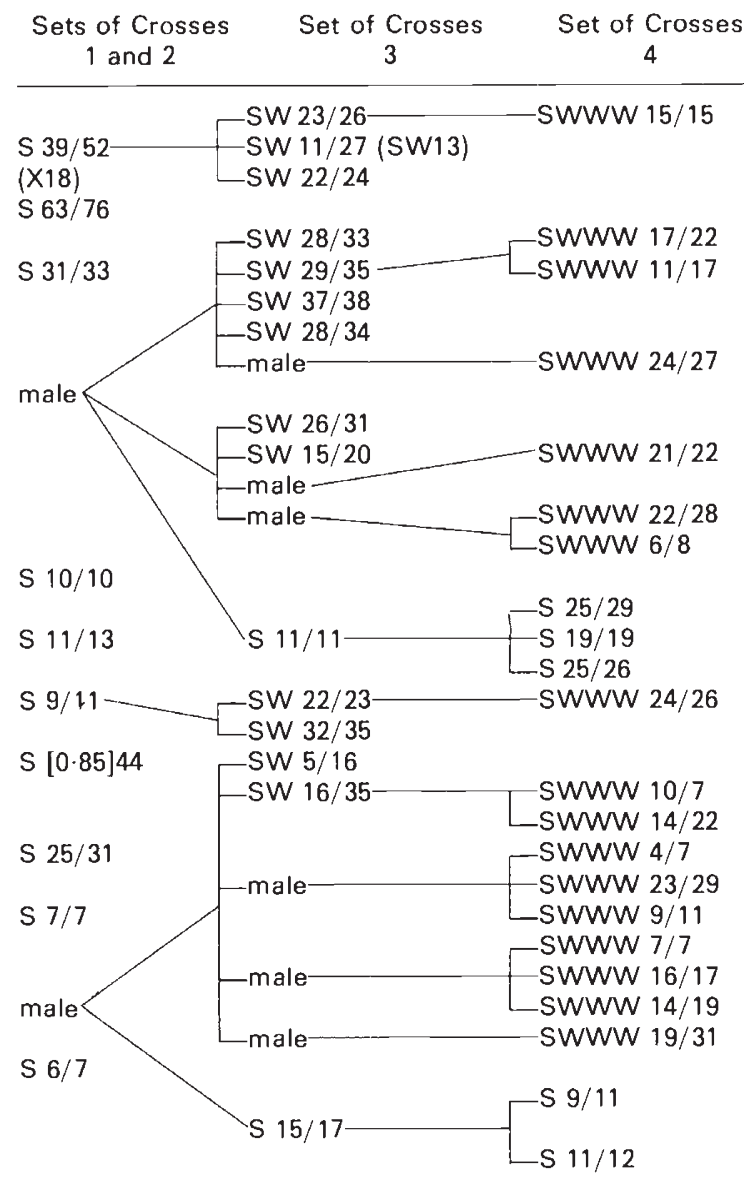

$S[0 \cdot 78] 20$

Figure 1 The relationships between females in the experiment. Each is shown as a code together with the number of $B$ containing eggs (top line) and the total number of eggs karyotyped (lower line). Code "S" denotes a purebred animal of Shakers Furze stock; "SW" denotes the offspring of the first cross to Winterton; "SWWW" those of the second. The number in square brackets following some individuals is a maximum likelihood estimate of transmission rate based on the models in Shaw (1984). The equivalent sample size follows it. Full sibships are traced separately from a common male parent. The two individuals followed by a code in brackets are referred to in the text
Figs. 1 and 2 summarise both the design and the results, showing the number and classes of crosses made, and the relationships between generations. From the figures it is apparent that the effect of the Shakers Furze and Winterton X-chromosomes could in principle be estimated. In view of the small numbers of crosses involved and the analyses described later, we did not follow this possibility up.

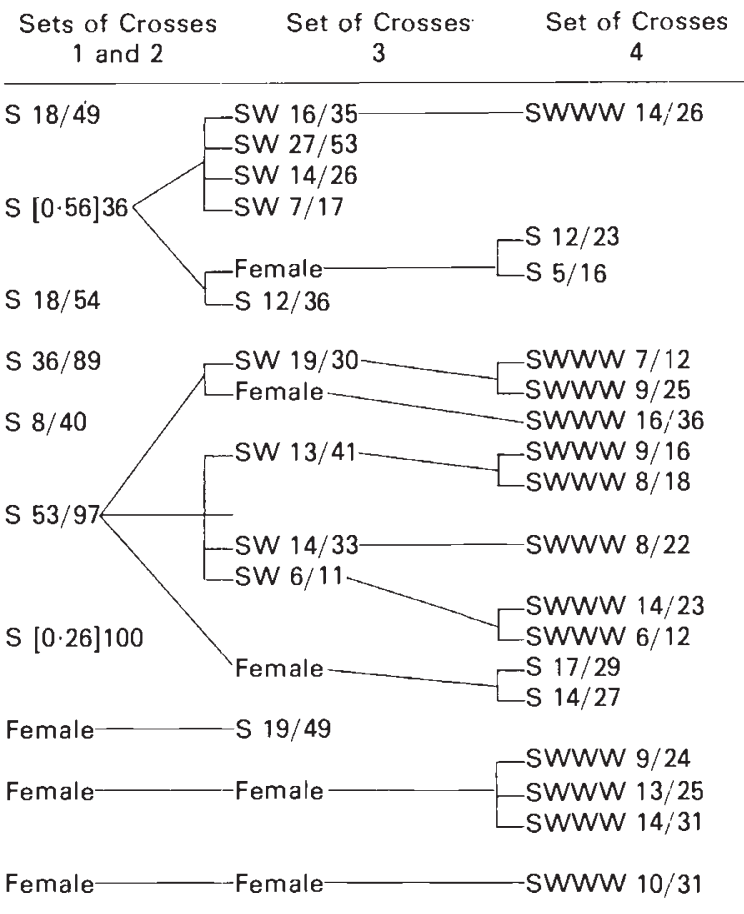

Figure 2 The relationships between males in the experiment, and the data from them. Symbolism is as for fig. I

\section{RESULTS}

The analysis of the results was carried out in a generalised linear regression framework; it was assumed that the logit of the observed transmission was linearly related to factors of interest. A good brief description of the technique can be found in Baker and Nelder (1978) (this reference refers to the package GLIM: we used similar facilities in GENSTAT). One result of this analysis is a number, the deviance, which is analogous to the mean square in a conventional analysis of variance. A smaller deviance indicates a better fit. If a model adequately explains the data, the deviance, in large samples, should be a random variable drawn from a chi-square distribution; chi-square may therefore be used as a test of goodness of fit. 


\section{Females}

There are five factors of interest which might explain variation in transmission rate between females in the crosses, and which can be estimated in the experiment. These are the effects of different B's; the additive effect of the backcross; the dominance effect of the backcross; other additive genetic effects (i.e., differences between families not attributable to the backcross); and consistent differences between generations. For the purpose of model fitting the "other additive genetic effects" were divided into those attributable to the ancestor in the first generation ("grandparental" effects in the backcross generation), and those attributable to an ancestor in the " $F_{1}$ " generation and therefore nested within the first category. The way in which the drive expected for particular individuals was worked out is shown in fig. 3.

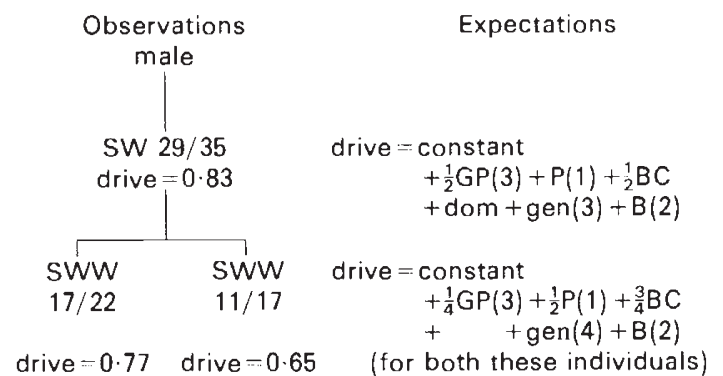

Figure 3 An example of how the expected values for individuals in the experiment were composed. The family tree is extracted from fig. 1; next to each individual are shown the components making up its expected value. $G P(3)$ refers to the effect of the third grandparent; $P(1)$ to the effect common to the family shown; $B C$ to the slope of the regression of drive on proportion of the genome made up of Winterton genes; dom to deviations of this regression from linearity, and gen(3) effects attributable to the particular batches of crosses of which these animals were part

The results are set out in table 1 . The drive rate is very far from being homogeneous (line 1), but none of the factors suggested above account wholely for the heterogeneity. Lines 7, 8, and 9 suggest that perhaps there are real differences between B's, between genotypes apart from the $B$ 's, and a real effect of the backcross. (The F-tests in the final column need to be interpreted very cautiously, as noted in the table heading). Looking further, however, the backcross effect is no longer apparent once the other two factors have been fitted (lines 16, 17); and the B-chromosome and other genetic effects are more or less confounded with one another. To add to the confusion, if the
Table 1 Analysis of deviance of the data on female transmission. In lines $1-6$ the deviance is tested against the chi-square distribution. In lines $7-18$ the reductions in deviance produced by the various factors are tested against the F-distribution. The reduction in deviance per degree of freedom is divided by the residual deviance per degree of freedom of the best fitting model found (analogously, in analysis of variance, the mean square for an effect is divided by the "error mean square"). This test should be viewed with considerable caution since it is based on the assumption that the denominator is a chi-square distributed variable-which is here known to be false. See Baker and Nelder, 1978 for further comments The notation $A / B$ means "the effect of $A$ after fitting $B$ ".

\begin{tabular}{|c|c|c|c|c|}
\hline Line & Factors in model & $\begin{array}{l}\text { Residual } \\
\text { deviance }\end{array}$ & df & Comments \\
\hline 1 & $\begin{array}{l}\text { None: overall } \\
\text { mean only }\end{array}$ & $156 \cdot 7$ & 47 & $P<10^{-10}$ vs. $X^{2}$ \\
\hline 2 & B-chromosomes & $113 \cdot 9$ & 43 & \\
\hline 3 & $\begin{array}{l}\text { Additive } \\
\text { genetic effects }\end{array}$ & $70 \cdot 7$ & 36 & $P=0.001$ vs. $X$ \\
\hline 4 & Backcross & $147 \cdot 8$ & 46 & \\
\hline 5 & Dominance & $152 \cdot 8$ & 46 & \\
\hline 6 & Generation & $154 \cdot 3$ & 44 & \\
\hline
\end{tabular}

\begin{tabular}{|c|c|c|c|c|}
\hline & Effect & df & $\begin{array}{l}\text { accounted } \\
\text { for }\end{array}$ & Comments \\
\hline 7 & $\begin{array}{l}\text { B-chromosomes } \\
\text { (B) }\end{array}$ & 4 & $42 \cdot 6$ & $P<0.05$ vs. F \\
\hline 8 & $\begin{array}{l}\text { Additive } \\
\text { genetic effecels } \\
\text { (GPP) }\end{array}$ & 11 & $86 \cdot 0$ & $P<0.001$ vs. $F$ \\
\hline 9 & $\begin{array}{r}\text { Backeross } \\
\left(B C^{\prime}\right)\end{array}$ & 1 & 8.9 & $\mathrm{P}<0.05$ vs. $\mathrm{F}$ \\
\hline 10 & Dominance & 1 & $3 \cdot 9$ & $\mathrm{P}>0.1$ vs. $\mathrm{F}$ \\
\hline 11 & Generation & 1 & $2 \cdot 4$ & $P>0.1 \mathrm{vs} . \mathrm{F}$ \\
\hline 12 & $\mathrm{~B} / \mathrm{GPP}$ & 4 & $3 \cdot 3$ & \\
\hline 13 & $B / B C:$ & 4 & $36 \cdot 8$ & \\
\hline 14 & GPP/B & 11 & $46 \cdot 7$ & \\
\hline 15 & GPP/BC & 11 & $77 \cdot 2$ & $P<0.01$ vs. $F$ \\
\hline 16 & $\mathrm{BC} / \mathrm{GPP}$ & 1 & $0 \cdot 0$ & \\
\hline 17 & $B C / B$ & 1 & 2.9 & \\
\hline 18 & $\mathrm{DOM} / \mathrm{B}$ & 1 & $10 \cdot 1$ & $P<0.05$ vs. $F$ \\
\hline
\end{tabular}

$\mathrm{B}$ effect is accepted, a significant dominance effect (i.e., non-linearity in the regression of transmission on generation) appears, although the backcross effect has become non-significant (lines 17, 18).

The confusion can be resolved by a harder look at the raw data. There are a number of very low transmission rates among these, and most of these appear in a single family tree, with an isolated instance, SW13, in a smaller tree (fig. 4). This suggests a genetic polymorphism in transmission rate among the individuals.

If such a genetic polymorphism does not exist, the individuals with lowest drive rates should be randomly distributed among all those measured, with no particular relationships between them. We tested this in the following way. Consider only the seven lowest individuals, those shown in fig. 4 . The average relatedness between any two of these individuals is 0.084 . We then calculated the distribution of average relatedness among sets of seven 


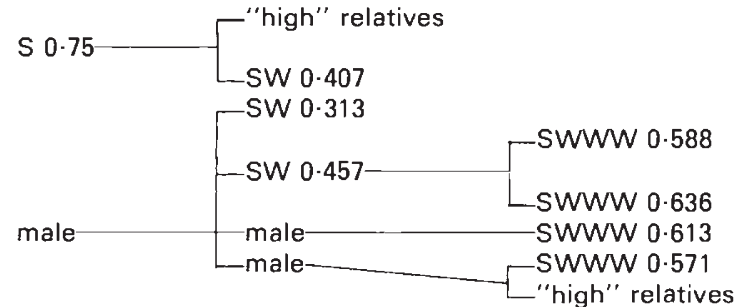

Figure 4 The relationships among the 7 crosses with least meiotic drive

individuals picked at random from a tree with the structure of relationships shown in fig. 1. Of 10,000 sets examined (out of the c. 60 million possible), only seven had an average relatedness greater than $0 \cdot 084$; a significance level of $0 \cdot 1$ per cent.

Splitting the dataset into two, by introducing a factor separating the seven lowest individuals from the remainder, explains almost all the heterogeneity in the data (table 2, line 2). Backcross, other genetic, or B-chromosome effects do

Table 2 The effect of including the post-hoc classification of individuals into high-drive or low-drive groups. The notation is as in table 1

\begin{tabular}{|c|c|c|c|c|}
\hline Line & Factors in model & $\begin{array}{l}\text { Residual } \\
\text { deviance }\end{array}$ & $\mathrm{df}$ & Comments \\
\hline 1 & $\begin{array}{l}\text { None: overall } \\
\text { mean only }\end{array}$ & $156 \cdot 7$ & 47 & \\
\hline \multirow[t]{2}{*}{2} & High-low drive $(\mathrm{H})$ & $68 \cdot 2$ & 46 & $P=0.02$ vs. $X^{2}$ \\
\hline & Effect & \multicolumn{2}{|c|}{$\begin{array}{l}\text { Deviance } \\
\text { accounted }\end{array}$} & Comments \\
\hline 3 & $\mathrm{GPP} / \mathrm{H}$ & $18.9)$ & & \\
\hline 4 & $\mathrm{BC} / \mathrm{H}$ & $0.1\}$ & & not significant \\
\hline 5 & $\mathrm{~B} / \mathrm{H}$ & $3 \cdot 8$ & & \\
\hline
\end{tabular}

not appear to contribute to the deviance once the polymorphism factor has been fitted (lines 3-5). The varied success of backcross and B-chromosome effects in reducing deviance in table 1 can be explained most easily in terms of the greater number of "low" individuals in the later generations of the crossing programme, and their close relationships. Similarly, the reduction in deviance produced by fitting a "dominance" factor after a factor allowing for differences between B's (table 1, line 18) can be attributed to the higher proportion of "low-drive" individuals found in the second generation of the programme.

By contrast, it is possible that real genetic differences exist between families, since their inclusion in the model allows a substantial, though not significant, improvement in fit (table 2, line 3 ). The remaining heterogeneity is presumably caused by non-additive genetic differences, minor environmental effects, and other factors not tested in this experiment.

So, to sum this up: the female drive data suggest the segregation of a simple genetic factor governing "high" or "low" drive. They do not suggest any large polygenic differences between the two populations tested. Within the sensitivity of the experiment, there appear to be no differences between the drive rates experienced by different B-chromosomes, nor any differences induced by unintended environmental variation between the generations.

\section{Males}

The same factors as for the females were used to investigate the male data. There is partial confounding between B and "other genetic effects", because no relatives of one family were measured in the first two generations. This means that fitting a family effect is identical to fitting the effect of the B common to the three individuals. However, this does not greatly complicate the interpretation of the results.

Table 3 Analysis of deviance of the data on male transmission. Notation is as in table 1 . The discrepancies in degrees of freedom between lines 7 and 14 and 8 and 12 are caused by the partial confounding mentioned in the text

\begin{tabular}{|c|c|c|c|c|c|}
\hline Line & Factors in model & \multicolumn{2}{|c|}{$\begin{array}{l}\text { Residual } \\
\text { deviance }\end{array}$} & $\mathrm{df}$ & Comments \\
\hline 1 & $\begin{array}{l}\text { None: overall } \\
\text { mean only }\end{array}$ & \multicolumn{2}{|l|}{$68 \cdot 9$} & 34 & $P=0.0004$ vs. $X^{2}$ \\
\hline 2 & B-chromosomes & \multirow{2}{*}{\multicolumn{2}{|c|}{$\begin{array}{l}46 \cdot 0 \\
35 \cdot 3\end{array}$}} & 31 & $P=0.04$ vs. $X^{2}$ \\
\hline 3 & $\begin{array}{l}\text { Additive } \\
\quad \text { genetic effects }\end{array}$ & & & 26 & $P=0.1$ vs. $X^{2}$ \\
\hline 4 & Backcross & \multicolumn{2}{|l|}{$65 \cdot 8$} & 33) & \\
\hline 5 & Dominance & \multirow{2}{*}{\multicolumn{2}{|c|}{$\begin{array}{l}66 \cdot 3 \\
59 \cdot 7\end{array}$}} & $33\}$ & P small of. line 1 \\
\hline \multirow[t]{2}{*}{6} & Generation & & & 32) & \\
\hline & Effects & \multicolumn{3}{|c|}{$\begin{array}{l}\text { Deviance } \\
\text { accounted } \\
\text { for }\end{array}$} & \\
\hline 7 & $\begin{array}{l}\text { B-chromosomes } \\
\text { (B) }\end{array}$ & 3 & $22 \cdot 9$ & & \\
\hline 8 & $\begin{array}{l}\text { Additive } \\
\text { genetic effects } \\
\text { (GGP) }\end{array}$ & 8 & $33 \cdot 5$ & & \\
\hline 9 & $\begin{array}{r}\text { Backcross } \\
(\mathrm{BC})\end{array}$ & 1 & $1 \cdot 5$ & & \\
\hline 10 & $\begin{array}{l}\text { Dominance } \\
\text { (I) }\end{array}$ & 1 & $1 \cdot 2$ & & \\
\hline 11 & $\begin{array}{c}\text { Generation } \\
\text { (G) }\end{array}$ & 1 & $2 \cdot 5$ & & \\
\hline 12 & $\mathrm{GPP} / \mathrm{B}$ & 7 & $11 \cdot 9$ & & \\
\hline 13 & $\mathrm{BC} / \mathrm{B}$ & 1 & $0 \cdot 6$ & & \\
\hline 14 & $\mathrm{~B} / \mathrm{GPP}$ & 2 & $1 \cdot 2$ & & \\
\hline 15 & $\mathrm{BC} / \mathrm{GPP}$ & 1 & $3 \cdot 4$ & & \\
\hline 16 & $\mathrm{D} / \mathrm{GPP}$ & 1 & 0.0 & & \\
\hline 17 & G/GPP & 2 & $5 \cdot 0$ & & \\
\hline 18 & GP & 2 & 17.5 & & \\
\hline 19 & $\mathrm{P} / \mathrm{GP}$ & 6 & $16 \cdot 1$ & & \\
\hline
\end{tabular}


The analysis is summarised in Table 3 . There is a good deal of heterogeneity among the crosses (line 1); this is substantially removed by fitting B effects or additive genetic effects (lines 2 and 3,7 and 8). Neither of the two backcross factors improve the fit more than fractionally (lines 4,5 , $9,10)$, but there is a hint of systematic differences between generations (line 6,11 ).

After fitting $B$ effects none of the other factors significantly improves the fit, whether judged against $\chi^{2}$ or by the deviance ratio (e.g., lines 12 , 13). On the other hand, after fitting additive genetic effects, both generation and backcross substantially, though not significantly, improve the fit (lines 15, 17). If the additive genetic effects are split into "grandparental" and other effects (see lines 18,19 above) it is apparent that the most significant component is the grandparental effectthe difference between the two major lineages in fig. 2. On fitting this, a linear trend is apparent in the residuals, (fig. 5) suggesting that a $\mathrm{B}$ effect, similar in each generation, is a better explanation

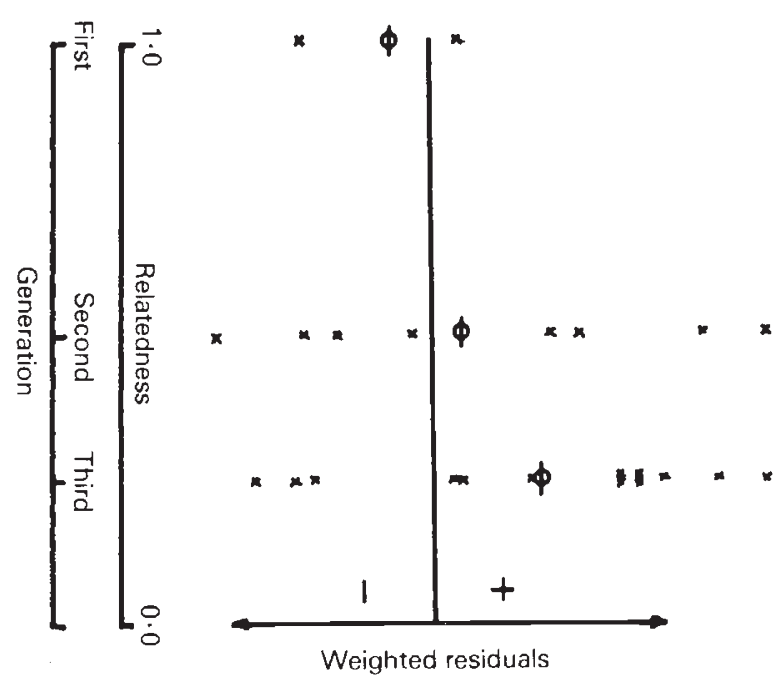

Figure 5 The weighted residuals after fitting grandparental effects to the male data plotted against the relatedness of the individuals to the "grandparents"- that is, the multiplier of the grandparental effect used in each generation. $\theta$ denotes the mean in each generation

of the data than the decreasing difference implied by an additive genetic effect. This trend in the residuals, of course, accounts for the improvement in fit produced by the "generation" and "backcross" factors seen in lines 15 and 17.

Thus, although one cannot make any very firm conclusions, it does seem that individual Bchromosomes tend to differ systematically in the transmission rate they manifest in males. There is no evidence for any large systematic differences between the populations, nor did any slight environmental differences between generations have any effect large enough to be detected on the average meiotic transmission rate.

\section{DISCUSSION}

The results demonstrate the segregation of a modifier gene in the female crossing programme, but do not suggest any polygenic differences between the two populations in male or female B-chromosome transmission rates. We can ask whether the modifier might account for the absence of the B from the Winterton population. In fact, this seems unlikely. There are a number of reasons why. The modifier appears to be present in all the populations in which drive and drag have been measured (Shaw, 1981 and manuscript in prep.). Thus, it is unlikely to be the immediate reason why the $\mathrm{B}$ is not found in some areas of Britain. It might, of course be a link in a chain of causation: for example, in colder areas its effect on drive or drag might be altered so that the equilibrium Bfrequency was zero or so small that the $B$ was lost by drift. However, there is at present no evidence to support such complex hypotheses. As mentioned in the introduction there is evidence that temperature could act directly on male transmission (Shaw and Hewitt, 1984). It also seems that the distribution of B's in East Anglia, at least, is not at equilibrium, (Shaw, 1983) so that an explanation of the distribution, other than an historical one, is inappropriate: in this case, one would certainly not expect to find any genetic barrier to the presence of the B at Winterton. The B might simply not have spread there yet. In the light of the experiments reported here, perhaps the most we can say about the causes of the geographic variation seen in the B-frequency of $M$. maculatus is that genetic differences in transmission rate among populations seem the least likely of the possible causes investigated so far.

Harvey (1977) suggested that there might be two genetic types of $M$. maculatus, one capable of supporting the $\mathrm{B}$, the other not, separated by a hybrid zone. This hypothesis has a number of difficulties: for example it forces the explanation of the overall distribution of B's back to an explanation of the distribution of the two types of grasshopper, which is not necessarily easier. The current experiments more or less contradict this notion since there was no evidence of hybrid unfitness 
between the populations crossed. Indeed, if anything, the crosses showed hybrid vigour, the " $F_{1}$ ", for example, developing to adult up to two days faster than either parent population.

The modifier polymorphism is of considerable interest in its own right. Models of the maintenance of the B-chromosome in $M$. maculatus populations (Shaw, 1984) suggest that its presence in a population imposes a genetic load. Genes tending to reduce this load will tend to increase in frequency: modifiers of transmission fall into this category. However, as B-frequencies fall, the selection pressure on the modifiers will tend to fall rapidly. The process is explored quantitatively in Shaw (1984): at the sort of B-frequency encountered in nature selection pressures on modifiers would be less than 1 per cent or so. It is unlikely that a modifier would be completely neutral in its other effects on the grasshopper. Any adverse effects would cause an equilibrium to be reached, at the frequency of the modifier where the selective advantage accruing from reduction in B-frequency among an individual's offspring just balances the intrinsic disadvantage of the modifier. This equilibrium frequency need not be very high.

If the modifier has dominant action in lowering transmission, the examples of it in the experiment are likely to have derived at least in part from the Winterton population, since X18 showed drive at the lower end of the "high" range, but produced, after ćrossing to a Winterton male, the unambiguously "low" individual SW13. (See fig. 1). In this case, we can say nothing about îhe presence of the modifier in the original Shakers Furze stock. However, as it seems to be present in other populations with B's (Shaw, 1981 and ms in prep.) it is very likely to be present at Shakers Furze. On the other hand, if the modifier is recessive, the same cross implies that it is present in both stocks. The conclusion must be that the modifier gene is present as a polymorphism in all $M$. maculatus populations. In this case the presence of the $\mathrm{B}$ in the population must simply increase somewhat the equilibrium frequency at which the modifier is maintained.

It is striking that the three lowest drive crosses occur in the $F_{1}$ generation. We have no solid explanation for this, although numerous hypotheses are possible. For example, the effect of the modifier might be more distinct on the Shakers Furze genetic background, as a result of selection like that described above, acting, perhaps, on very many genes of very slight effect (cf Shaw 1984, section 5).

The polymorphism is one of rather few of this general class demonstrated in B-chromosome sys- tems. The results of Teoh and Jones (1978) in rye have been mentioned. Other examples refer more to the B-chromosome itself. In maize a complex of loci on the B itself can modify the directed nondisjunction that leads to meiotic accumulation in this species (Review in Carlson, 1978). As maize occurs only in cultivation, the natural evolutionary processes involved in this have not been studied. Likewise, Subba Rao (1980) showed that in Pearl Millet similar karyotypic mutations of the B showed similar changes in transmission frequency-here, apparently, independently of the parental genome.

In some ways the results of investigations on dipteran "segregation distorter" and "sex-chromosome distorter" loci are more directly related to the polymorphism discussed here, as the evolutionary dynamics of the systems are more fully worked out. Segregation distorter in Drosophila melanogaster can serve as an example. Hiraizumi and Hartl (1977) provides a general review. A detailed theoretical model is given in Charlesworth and Hartl (1978). The cases examined are in a sense complementary to those looked at in Shaw (1984), despite the differences in the systems. Shaw (1984) considered the progress through a populaion of an allele reducing drive and showed that an equilibrium would result if the allele had any deleterious pleiotropic effects, as mentioned above. On the other hand, Charlesworth and Hartl showed that such an interior equilibrium did not exist for an unlinked neutral modifier. (Even if the modifier is not neutral, the interior equilibrium can only be reached from the margin where the reducing allele is fixed through the initial increase of the enhancing allele to some critical frequency, followed by invasion of the driven allele once the average drive it experiences outweighs its fitness disadvantages. The path to equilibrium is more obvious starting from the other margin, fixed for the enhancer). The analogies to the polymorphisms found by Charlesworth and Hartl and other authors, involving close linkage, would presumably involve variant $\mathrm{B}$ chromosomes with reduced drive (and here the male results of the present experiment may be of interest) but reduced selective disadvantage in $2 \mathrm{~B}$ individuals. The fate of such variants has not been examined. Overall, however, the broad conclusion must be that there is ample scope for continued stable polymorphism in systems involving meiotic drive.

To sum up, results of this experiment are evidence of the complexity of the genetic structure in one particular wild population. They are striking illustrations of the power of natural selection; but 
they also illustrate that natural selection does not always lead to the results that might be adaptively optimal for the species: in this case, total elimination of the B. Finally, they provide an insight into how an organism may respond to one particular form of "selfish DNA".

Acknowledgements MWS is grateful to both the Natural Environment Research Council and the University of East Anglia for financial support during this work. Range Control, Stanford Battle Training Area kindly gave permission to collect grasshoppers from Shakers Furze.

\section{REFERENCES}

BAKER, R. I. AND NELDER, I. A. 1978. The GLIM system, release 3. Royal Statistical Society, London.

BARKER, J. S. F. 1966. Climatological distribution of a grasshopper supernumerary chromosome. Evolution, 20, 665-667.

CARlson, w. R. 1978. The B-chromosome of corn. Ann. Rev. Genet., 12, 5-23.

CHARLESWORTH, B. ANI) HARTL, D. L. 1978. Population dynamics of the segregation distorter polymorphism of Drosophila melanogaster. Genetics, 89, 171-192.

HARTL, D. L. AND HIRAIZUMI, Y. 1976. Segregation distortion in Genetics and Biology of Drosophila, vol. $1 \mathrm{~b}$ Ashburner M. and Novitski E. (eds.) Academic Press New York.

HARVEY, A. R. 1977. The expression of supernumerary chromo somes in Myrmeleotettix maculatus (Thunb.) (Acrididae) Ph.D. Thesis, University of East Anglia.

HEWITT, G. M. 1973. The integration of supernumerary chromosomes into the orthopteran genome. Cold Spring Harbour Symp. Quant. Biol., 38, 183-194.
HEWITT, G. M. AND JOHN, B, 1967. The B-chromosome system of Myrmeleotetix maculatus. III. The Statistics. Chromosoma, 21, 140-162.

HEWITT, G. M. AND RUSCOE, C. N. F. 1971. Changes in microclimate correlated with a cline for B-chromosomes in the grasshopper Myrmeleotettix maculatus. J. Anim. Ecol., 40, $753-765$

JONES, N. AND REES, H. 1983. B-chromosomes, Academic Press, London.

KELLY-STEBBiNGS, A. AND HEWITT, G. M. 1972. The laboratory breeding of British Gomphocerine grasshoppers (Acrididae: Orthoptera). Acrida, 1, 233-245.

PROUT, T., BUNDGAARD, J. AND BRYANT, S. 1973. Population genetics of modifiers of meiotic drive. I. The solution of a special case and some general implications. Theor. Pop. Biol., 5, 446-465.

RAMEL, C. 1980. A B-chromosome system of Myrmeleotettix maculatus (Thunb.) (Orthoptera: Acrididae) in Sweden. Hereditas, 92, 309-313.

SHAW, M. W. 1981. A B-chromosome cline in the Mottled Grasshopper. Ph.D. Thesis, University of East Anglia.

SHAW, M. W. 1983. Rapid movement of a B-chromosome frequency cline in Myrmeleotetix maculatus. (Orthoptera: Acrididae). Heredity, 50, 1-14.

SHAW, M. W. 1984. The Population genetics of the B-chromosome of Myrmeleotettix maculatus (Thunb.). (Orthoptera: Acrididae). Biol. J. Linn. Soc., 23, 77-100.

SHAW, M. W. AND HEWIT, G. M. 1984. The effect of temperature on meiotic transmission rates of the B-chromosome of Myrmeleotettix maculatus (Orthoptera: Acrididae). Heredity, 53, 259-268.

SUBBA RAO, M. K. 1980. Inheritance of B-chromosome in Pearl Millet. Heredity, 45, 1-6.

TEOH, S. B. ANI JONES, N. 1978. B-chromosome selection and fitness in Rye. Heredity, 41, 35-48. 